\title{
A nonblocking mechanism for regulating the transmission of network management polls
}

\author{
A. B. Bondi \\ AT\&T Labs \\ PO Box 3030 \\ Crawfords Corner Road \\ Holmdel NJ 07733-3030 \\ U.S.A. \\ Tel. +1908949 1921, Fax. +19089491720. \\ E-mail: andre.bondi@att.com
}

\begin{abstract}
We propose and analyse the performance of a new mechanism that allows an arbitrary number of network management polling requests to be outstanding at any instant, while preventing the flooding of the network with polling messages. An advantage of the mechanism over one previously described in the open literature is that it continues to function smoothly, without freezing for prolonged periods, even if many failed nodes are polled in succession.
\end{abstract}

\section{Keywords}

Network management polling, status polling, SNMP.

\section{INTRODUCTION}

In IP-based and SNMP-based network management systems, the status of managed nodes is ascertained by sending polling messages at scheduled times or on demand according to need (Rose, 1991). Care must be taken to ensure that bursts of polling messages do not interfere unduly with user traffic. On the other hand, because timely dispatch of polling messages is essential to network management, a polling dispatch mechanism should not prevent polls from taking place for any significant length of time. 
One way to reduce the risk that the user's network is flooded with polling messages is to restrict the number that may be unacknowledged at any instant (Sturm, 1995). This is done in such systems as HP OpenView Node Manager and implementations of OpenView on other systems, e.g., AT\&T OneVision. ${ }^{1}$ We shall refer to this method as the constrained outstanding poll method of degree $N$, or COP-N. ${ }^{2}$ In the implementations just mentioned, $N$ is set to three. Under COP-N, the rate at which polls are emitted is inversely proportional to the time taken to resolve a poll, because there is no explicit control on the polling rate. Therefore, the polling rate may be higher than desirable if the acknowledgment time is short and all polled nodes are responding. This is particularly noticeable when nodes are being discovered. As the network management system "learns" about nodes about which it has no record in its database, it must verify their status and existence by polling them, too. Polling is done by means of ICMP (internet control message protocol) ping messages. Polls may occur in bursts, especially when a network management system is being brought up for the first time (AT\&T/HP, 1994).

Suppose that the network management system allows unacknowledged polls to be outstanding to at most three nodes any instant. If the first attempted ping (ICMP message) to a node fails to elicit a response after a timeout interval of ten seconds, at most three subsequent attempts follow. The timeout interval associated with each attempt is set to twice that of the previous attempt. The sequence of attempts terminates if an acknowledgment to the current ping is received before a timeout expires, or after the timeout has expired on the fourth attempt. If no response has been received by the network management system after all four attempts, the node in question is declared to be inoperative, and the colour of the corresponding icon on the network management display is changed accordingly (Sturm, 1995). We call this sequence of attempts a polling cycle. The maximum total time between the beginning of the first attempt and the end of the fourth attempt is 150 seconds.

We have already seen that COP-N does not protect the network from bursts of polls when nodes are responsive. It has also been reported that COP-N may prevent polling activity when nodes are unresponsive (Sturm, 1995). Consider a scenario in which three failed or unreachable nodes are polled in rapid succession. Since no more than three nodes may have unacknowledged polls concurrently, it will be impossible to determine the status of any other node for the next 2.5 minutes. Similarly, five minutes will elapse before any polling can be done if six unresponsive nodes are polled in succession, 7.5 minutes if nine unresponsive nodes are encountered, etc (Sturm, 1995). If many nodes are unreachable, e.g.,

1. All information in this paper about the HP OpenView and AT\&T OneVision network management systems is contained in published sources. OpenView is a trademark of Hewlett Packard. OneVision is a trademark of AT\&T Corp. and of NCR Corp. (formerly known as AT\&T Global Information Solutions).

2. This term has not been used previously. 
because of a failed router, the time from status or configuration change to operator notification may be excessive.

One might wish to overcome the freezing of polling activity by increasing the number of outstanding polling messages. This would be ill advised. Apart from failing to prevent freezing if more than $N$ unresponsive nodes are polled in sequence, the increase in $N$ would allow bigger bursts of polls to be released, to the extent that acknowledgments might not be processed fast enough by the network management station (Sturm, 1995).

In this paper, we propose and model the performance of an alternate mechanism for allowing an arbitrary number of nodes to be polled concurrently without receiving acknowledgments. The mechanism prevents flooding with ping messages by ensuring that polling messages are transmitted at a rate that does not exceed a prescribed level. It might also be tuned to ensure that the kernel of the host operating system, which performs protocol handling in operating systems such as $\mathrm{UNIX}^{\mathrm{TM}}$, is not overtaxed by returning polling messages. It also ensures that polls of unresponsive nodes do not impede the monitoring of other nodes. We shall call this method of regulating the emission of polling messages the regulated poll emission method (RPE).

\section{DESCRIPTION OF THE RPE METHOD}

Status polling requests may be generated in bursts, depending on how they are scheduled. To prevent pings from being transmitted at an excessive rate, they should be scheduled for transmission in rapid succession at a controlled rate specified by the network administrator. One method of doing so is a modification of the leaky bucket algorithm similar to that proposed to regulate the transmission of ATM cells (Eckberg, Luan, \& Lucantoni, 1990). In this algorithm, a set number of pings could be transmitted within a specified time frame. Pings in excess of this number would be queued for later dispatch. ${ }^{3}$ Alternatively, pings would be queued for transmission a minimum time apart, thus reducing the burstiness of ping transmissions and capping the bandwidth they use. Under the RPE method, polling messages would be dispatched at intervals of length not less than some quantity $\tau$. Let us suppose that all polling messages have constant length, and that the time to emit each one is $C$, with $C<\tau$. Then the maximum poll transmission rate is simply $1 / \tau$, regardless of the states of nodes polled or the sequence in which they are polled.

To speed the processing of acknowledgments, records of unacknowledged pings would be registered in an ordered data structure indexed by target IP address.

3. Ping messages would never be discarded as might be the case with ATM cells, selectively or otherwise, because this could lead to a false diagnosis of node failure. 
To speed the management of timeouts, records of unacknowledged pings would be kept in another ordered data structure, indexed by the time at which a timeout is scheduled. Each record in one data structure would contain a pointer to its mate in the other, to facilitate rapid removal upon timeout or upon receipt of an acknowledgment, whichever occurred first.

The RPE method has the following advantages:

1. The method allows an arbitrary number of status polls to be outstanding simultaneously.

2. The method permits rapid update of status changes on operators' network maps.

3. The method prevents the release of bursts of status polling messages onto the network.

However, the method does have limitations also. While the RPE method would certainly regulate the transmission of outbound ICMP messages, there would not be any equivalent rate control for the reply (acknowledgment) messages. Under stable conditions, the inbound message arrival sequence would not be unlike that of the outbound sequence. However, we do not know if the echos sent in response to pings would arrive in a bursty manner, especially if they were collectively delayed at a (possibly) transient bottleneck on their return path.

In the next section, we shall model the duration of a status polling cycle. Then, we shall use this model to compare the throughput and delay characteristics of the COP-N and RPE methods.

\section{MODELING THE DURATION OF A STATUS POLL CYCLE}

The duration of a poll is determined by the number of polling attempts needed to determine if the polled node is reachable and responsive or either unreachable or unresponsive. In the proposed method, this duration is the amount of time during which a pending poll request must be stored. In the method described in (Sturm, 1995), this is the amount of time during which a dispatch slot (or queue in the terminology of (Sturm, 1995)), cannot be used to perform another poll.

Figure 1 shows the components of the duration of a polling cycle consisting of $K$ attempts. The duration of the $k$ th attempt $(1 \leq k \leq K)$, consists of the polling request emission time, $C$, and the lesser of the timeout interval $T_{k}$ and the time to receive an acknowledgment, $A_{k}$. The probability that the $k$ th polling attempt is unacknowledged is $p_{k}$, and that it was received before the timeout, $1-p_{k}$. In OpenView/OneVision, there are $K=4$ attempts, with $T_{1}=10$ seconds, $T_{2}=20$ seconds, $T_{3}=40$ seconds, and $T_{4}=80$ seconds. Since $\sum T_{i}=150$ seconds, 2.5 minutes may elapse from the time a node is polled to the time at which it is declared unreachable, or down. 


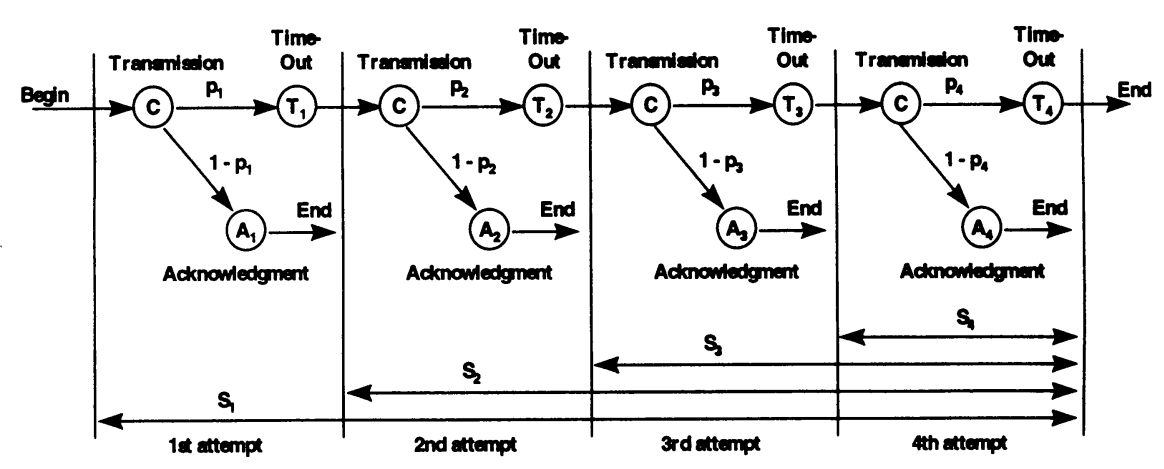

Figure 1 Components of a polling cycle with 4 attempts.

A polling attempt is unacknowledged if any of the following events occur:

1. An ICMP polling message is lost on its outward journey, say with probability $\gamma_{1}$.

2. A node might be down, or it might simply not be reachable because all paths to it are blocked for some reason, say with probability $\gamma_{2}$. For example, the node might only be reachable via a single bridge or router, which itself could be inoperative.

3. The corresponding acknowledgment packet might be lost on its return journey owing to network congestion, say with probability $\gamma_{3}$.

4. The acknowledgment does not return prior to the expiration of the current timeout, say with probability $\operatorname{Pr}\left(A_{k}>T_{k}\right)$.

It follows that $p_{k}$ is given by

$p_{k}=1-\left(1-\gamma_{1}\right)\left(1-\gamma_{2}\right)\left(1-\gamma_{3}\right) \operatorname{Pr}\left(A_{k}<T_{k}\right)$

provided all four events occur independently.

The acknowledgment return time $A_{k}$ is also influenced by the three factors affecting the $\gamma_{i}$ s. Because the length of $A_{k}$ depends on network topology as well as on congestion levels, determining the form of its distribution may be complex. For the purpose of illustration, we shall assume that $A_{k}$ is exponentially distributed with a mean equal to a round trip time commensurate with the network being managed. In a local area network, $E\left[A_{k}\right]$ might be less than $10 \mathrm{msec}$. For nodes that are further away, the round trip time may be much larger, e.g., 100-200 msec. Under severe congestion at the destination or intermediate nodes, $E\left[A_{k}\right]$ may even be of the order of a few seconds. 
Notice that if all nodes are unreachable or unresponsive, $\gamma_{2}=1$ and hence $p_{k}=1$. Similarly, regardless of the state of the nodes, if all ICMP packets are lost in either one or both directions, $\gamma_{1}=1$ or $\gamma_{3}=1$, and again $p_{k}=1$, as expected. Also, since the timeout interval $T_{k}$ doubles for successive values of $k$, the probability that the acknowledgment fails to arrive before timeout expiry diminishes, and the failure of the $k$ th polling attempt tends to be due more to either or both of the risks of packet loss or the unresponsiveness of the polled node. Finally, we verify the consistency of the equation by observing that if the timeout goes off immediately, i.e., if $T_{k}=0, \operatorname{Pr}\left(A_{k}<T_{k}\right)=0$ and $p_{k}=1$ as expected.

Equation (1) contains the implicit simplifying assumption that $p_{k}$ only depends on $k$ through the increasing timeout interval $T_{k}$. This is very likely to be true for the factors accounting for polling message loss, since congestion will fluctuate quickly relative to the lengths of the timeout intervals. If we had information about the likelihood of a node being unresponsive on one polling attempt given that no response was received in previous attempts, $\gamma_{2}$ could be made dependent upon $k$. However, responses might not be received for a variety of reasons. For example, the node might be switched off, in the course of being rebooted, or otherwise on the verge of restoration.

The value of the $\gamma_{i}$ s may vary with the sequence of nodes either currently scheduled for polling or for which polls have been initiated but not yet resolved. For instance, if a failed router lies on the unique path to a large number of managed workstations that are scheduled for polling within a short amount of time, the value of $\gamma_{2}$ will tend to one. On the other hand, if the router and all paths to it are fully operative, $\gamma_{2}$ will tend to zero.

To obtain upper bounds on the throughputs of either polling method, it is necessary to determine the mean duration of a polling cycle. We may model the duration of an entire polling cycle consisting of $K$ attempts as follows. Let $C$ denote the time to emit an ICMP polling message. Let $S_{k}$ denote the remaining time to resolve the poll at the beginning of the $k$ th attempt, i.e., the combined holding times of the $k$ th and subsequent attempts, if any. Then, $S_{K}$ is the time to resolve the final polling attempt of the cycle, while $S_{1}$ is the entire time to make the determination that the polled node is reachable or not. For attempts other than the $K$ th, a subsequent attempt is necessary with probability $p_{k}$ and is unnecessary with probability $1-p_{k}$. If a $(k+1)$ th attempt is not needed, the duration of the $k$ th attempt will be the time from emission to the return of the acknowledgment. If it is needed, the remainder of the polling cycle includes the $k$ th timeout and the duration of the $k+1$ attempt, $S_{k+1}$. Therefore,

$S_{k}=C+\left(1-p_{k}\right) A_{k}+p_{k}\left(T_{k}+S_{k+1}\right) \quad k=1,2, \ldots, K-1$.

At the $K$ th attempt, the remainder of the polling cycle lasts $A_{K}$ if the acknowledgment returns before the $K$ th timeout expires and $T_{K}$ otherwise. Hence, 
$S_{K}=C+\left(1-p_{K}\right) A_{K}+p_{K} T_{K}$.

By inspection, we see that the components of the average polling cycle time are given as follows:

1. The mean duration of the final stage of the polling cycle is given by

$E\left[S_{K}\right]=E[C]+\left(1-p_{K}\right) E\left[A_{K}\right]+p_{K} E\left[T_{K}\right]$.

2. The mean duration of the remaining time of the polling cycle at the beginning of the $k$ th attempt is

$$
\begin{aligned}
E\left[S_{k}\right] & =E[C]+\left(1-p_{k}\right) E\left[A_{k}\right] \\
& +p_{k}\left(E\left[S_{k+1}\right]+E\left[T_{k}\right]\right) \quad k=K-1, K-2, \ldots, 2,1 .
\end{aligned}
$$

The average duration of a polling cycle, $E\left[S_{1}\right]$, may be obtained by backward recursion beginning with $E\left[S_{K}\right]$.

Finally, we derive an approximate expression for the expected number of polling attempts in a cycle. We need this expression to determine the maximum expected bandwidth used for polling with either scheme. The expression is approximate because our formulation of it assumes that the events on successive polling attempts within a cycle are independent. They clearly are not, because if a node is inoperative or unreachable on one attempt it will almost certainly be unreachable on subsequent attempts. Under the assumption of independence, only one attempt is required in a cycle with probability $1-p_{1}$. Only two attempts are required with probability $p_{1}\left(1-p_{2}\right) . K$ attempts are required with probability $K-1$

$\prod_{i=1} p_{i}$. If $N_{P}$ denotes the number of polling attempts required within a cycle, under the assumption of independence,

$E\left[N_{P}\right]=\sum_{k=1}^{K-1} k\left(1-p_{k}\right) \prod_{j=1}^{k-1} p_{j}+K \prod_{k=1}^{K-1} p_{k}$

If $s_{p}$ denotes the size of a polling message, and $X$ denotes the maximum throughput under whichever polling dispatch mechanism is used, the maximum polling bandwidth demand is bounded above by $s_{p} X E\left[N_{P}\right]$.

\section{POLLING CYCLE THROUGHPUTS AND FREEZING}

\subsection{COP-N method}

For the COP-N method described in (Sturm, 1995), the maximum rate at which polling cycles may be initiated if at most $N$ polls are outstanding is 
$X_{N}=N / E\left[S_{1}\right]$.

Thus, the throughput will be driven down if the probability that an ICMP poll is acknowledged is small for whatever reason, i.e. if $p_{k}$ is close to 1 . The system will freeze for a period of at most $\sum_{i=1}^{K} T_{i}$ seconds, the sum of the timeout intervals, if $N$ unreachable nodes are polled in rapid succession.

If the network is functioning perfectly, the maximum throughput is given by

$X_{N}^{*}=N /(a+C)$

where $a$ is the mean acknowledgment time in the absence of failures or packet losses and $C$ is the time to emit an ICMP poll. If $a$ is small, bursts of polls may swamp the network at rates high enough to degrade application performance.

\subsection{RPE method}

Let $\tau$ be the minimum time between ICMP poll emissions. Then $1 / \tau$ is the maximum rate at which the network mangement engineer chooses to issue polling requests. We must have $\tau>C$ because of hardware and software limitations. However, we must also have $\tau<E\left[S_{1}\right] / N$ for this method to have a higher throughput than the COP-N method. Let $M$ be the maximum allowed number of nodes for which polling requests may be outstanding. The value of $M$ is equal to the maximum number of entries on a list of unacknowledged polls, and would be determined by memory constraints and/or by performance considerations. Typically, $M$ will be much larger than $N$.

The average number of outstanding polling requests resident in memory is equal to the product of the the rate at which polls are emitted and the average duration of a polling cycle, i.e., $X E\left[S_{1}\right]$. This number must be less than the maximum number of unresolved polling cycles, $M$. Hence, we must have $X E\left[S_{1}\right]<M$. But $X<1 / \tau$, so we finally have the following bounds on the node polling rate:

$X<\min \left[1 / \tau, M / E\left[S_{1}\right]\right]$

Of these, $\tau$ is a tunable parameter whose value would be determined by network and operating system constraints, $C$ is determined by the polling message size and the bandwidth of the subnetwork closest to the network management workstation, while $M$ is a tunable parameter whose value need only be restricted if memory is scarce. Under normal operating conditions, $\tau$ would be the parameter controlling the polling rate. The polling would only be constrained by $M$ if $E\left[S_{1}\right]$, the average duration of a polling cycle, were to become very large for any reason. $M$ should be made large enough to allow a polling rate of $1 / \tau$ under normal conditions. Any of the following factors could limit the (desired) polling rate: 
1. the need to keep the polling rate down to avoid saturating the operating system in the network management station, thus necessitating an increase in $\tau$,

2. the need to keep the polling rate down to avoid saturating the managed network with polling traffic, again necessitating an increase in $\tau$,

3. very long propagation delays or network congestion, resulting in long acknowledgment return times, and hence a large value of $E\left[S_{1}\right]$,

4. a high incidence of node failure, again resulting in a large value of $E\left[S_{1}\right]$.

If the first two constraints were loose, $M$ would have to be made suitably large to sustain throughput. Conversely, if either of the first two constraints were tight, the value of $M$ would not be critical. On the other hand, a large value of $M$ would ensure that polling would continue without interruption even if a large number of failed nodes were encountered in succession. Polling would never be frozen unless $M$ unreachable nodes were polled in rapid succession. In the limiting case, $M$ would never be an effective constraint if it were greater than or equal to the number of nodes under management or the number of nodes that could be discovered. If freezing did occur under this method, it could be indicative of a very serious failure in the network, e.g., it could indicate the failure of a router or bridge that is the sole means of accessing the nodes in question. By contrast, in the COP-N method with $N<<M$ polls outstanding, the polling process would freeze as soon as $N$ failed or unresponsive nodes were polled in succession. On the other hand, if both nodes and network are reliable, COP-N may (perhaps intermittently) swamp the network with polling messages, while RPE could be tuned to prevent this from happening by choosing an appropriate value for $\tau$.

\section{DELAY ANALYSIS}

A polling dispatch mechanism should be able to emit polls in a timely manner once they have been scheduled, whether or not the nodes to be polled are reachable. In this section, we compare the delay characteristics of the proposed rate control mechanism with those of the limited slot mechanism. Because polls may sometimes occur in bursts or else infrequently (Sturm, 1995; AT\&T/HP, 1994), we shall not attempt to perform steady-state queueing analyses of the two polling mechanisms. Instead, we shall perform an analysis of the behaviour of the two mechanisms when a fixed number of polls is scheduled for dispatch, of which a given number of unreachable or failed nodes will be polled consecutively.

Suppose that $n>N$ nodes are scheduled for dispatch at time $t$, and that of these, the first $N$ nodes are either down or otherwise unreachable, while the states of the other $n-N$ nodes are unknown. Suppose further that $M$, the maximum permissible number of initiated unresolved polling cycles under RPE, is much greater than the average number of nodes with initiated unresolved polling cycles, $E\left[S_{1}\right] / \tau$. 
Case 1: COP-N Method. The polling cycles for the $N$ failed nodes last $\sum_{k=1}^{K} T_{k}$ seconds. During this period, no other nodes can be polled. Then, we may expect that the status poll of the last node in the queue will be initiated at time $t+(n-N-1) E\left[S_{1}\right]+\sum_{k=1}^{K} T_{k}$ seconds, for $n \geq N+1$.

Case 2: RPE Method. Suppose that there is no effective limit on the number of unresolved polling requests, i.e., that $M$ is very large. If $n(<M)$ nodes are scheduled for polling at time $t$, and the minimum time between polls is set to be $\tau$, the $n$th polling cycle will be initiated at time $t+(n-1) \tau$ seconds, whether or not the first $N$ nodes to be polled are unreachable. Moreover, the $n$th polling cycle will be initiated at time $t+(n-1) \tau$ regardless of which or how many of the $n-1$ nodes polled before it are unreachable. This shows the robustness of the method under adverse conditions.

For the specified scenario, the delay performance will be better with the rate controlled mechanism if $\tau$ is set so that

$(n-1) \tau \leq(n-N-1) E\left[S_{1}\right]+\sum_{k=1}^{K} T_{k}$

In particular, for $n=N+1$, i.e., for the node queued just behind the first $N$ at time $t$, the $n$th polling cycle will be initiated earlier with the rate controlled mechanism provided that

$\tau \leq \frac{\sum_{k=1}^{K} T_{k}}{N}$

Since $\tau$ will typically be on the order of seconds or a fraction of a second, while the sum of the timeout intervals is 150 seconds, the advantage of the rate controlled mechanism is clear, especially if it is quite likely that nodes will be unresponsive for any reason.

\section{NUMERICAL ILLUSTRATIONS}

\subsection{Analysis of numerical results}

To illustrate our performance model, we shall compare throughput bounds and sample delays for the COP-N method and the RPE method under different scenarios. We make the following assumptions:

1. The acknowledgment return time $A_{k}$ is exponentially distributed with a mean that is independent of the polling attempt in the cycle. The mean depends on the network topology and traffic conditions in a manner that we shall not attempt to capture. For a network with remote nodes, this mean might be 
100 milliseconds. For a local area network, it might be as low as 2 milliseconds.

2. The default size of a polling message (ping) is 64 bytes.

3. The bandwidth of the local area network to which the network management station is attached is 10 Mbps.

4. At most four polling attempts are made within a polling cycle. The length of the $k$ th timeout is $10 \times 2^{k-1}$ seconds, for $k=1,2,3,4$.

5. Unless otherwise stated, under the RPE method, the maximum number of allowed outstanding polls is effectively infinite.

The effects of different parameter values may be easily determined by coding the formulae in a spreadsheet, as illustrated in Table 1.

As shown in Figure 2, the mean number of polls per polling cycle (marked on the left vertical axis) ranges from an inherent minimum of one to the programmed maximum of four as the probability of polling an unresponsive node increases. This quantity is only mildly dependent on the ping return time. The mean polling cycle duration (marked on the right vertical axis) ranges from a small level to the sum of the timeout intervals as the probability of polling an unresponsive node increases from zero to one. The time is shorter when the average ping return time is shorter, as one might expect. However, this effect disappears when nodes are unresponsive, since the large timeout intervals become the dominant factor. Consequently, under COP-N, the rate at which nodes may be polled diminishes as the probability of polling an unresponsive node increases, while the number of polling attempts per cycle rises from one to four as one would expect. Because COP-N is almost frozen when nodes are unresponsive, the bandwidth due to polling under COP-N diminishes, too.

In contrast, as illustrated in Figure 3, under RPE, provided enough memory is allocated for outstanding polls, the maximum number of nodes polled per second is constant. The coincidence of the dashed and solid lines for RPE shows that the node polling rate is independent of the ping round trip time. To avoid reducing the node polling rate when the probability of polling many unresponsive nodes in sequence is close to one, one must store a number of pending polls equal to the sum of the timeout intervals multiplied by the maximum polling rate, $1 / \tau$. In Figure 3, this is reflected in the slight drop in the node polling rate when the maximum permitted number of outstanding polls $M$ is 1000 rather than 1500 .

As shown in Figure 4, the bandwidth required for polling increases somewhat under RPE as the probability of polling an unresponsive node increases, because the expected number of polls per node increases. When the probability of encountering an unresponsive node is low and the ping return time is low, COP-N may have a much higher node polling rate and consequent demand for bandwidth than is desired. Also, the node polling rate of RPE is insensitive to the presence of unresponsive nodes. It is worth noting that the range of node polling rates and 


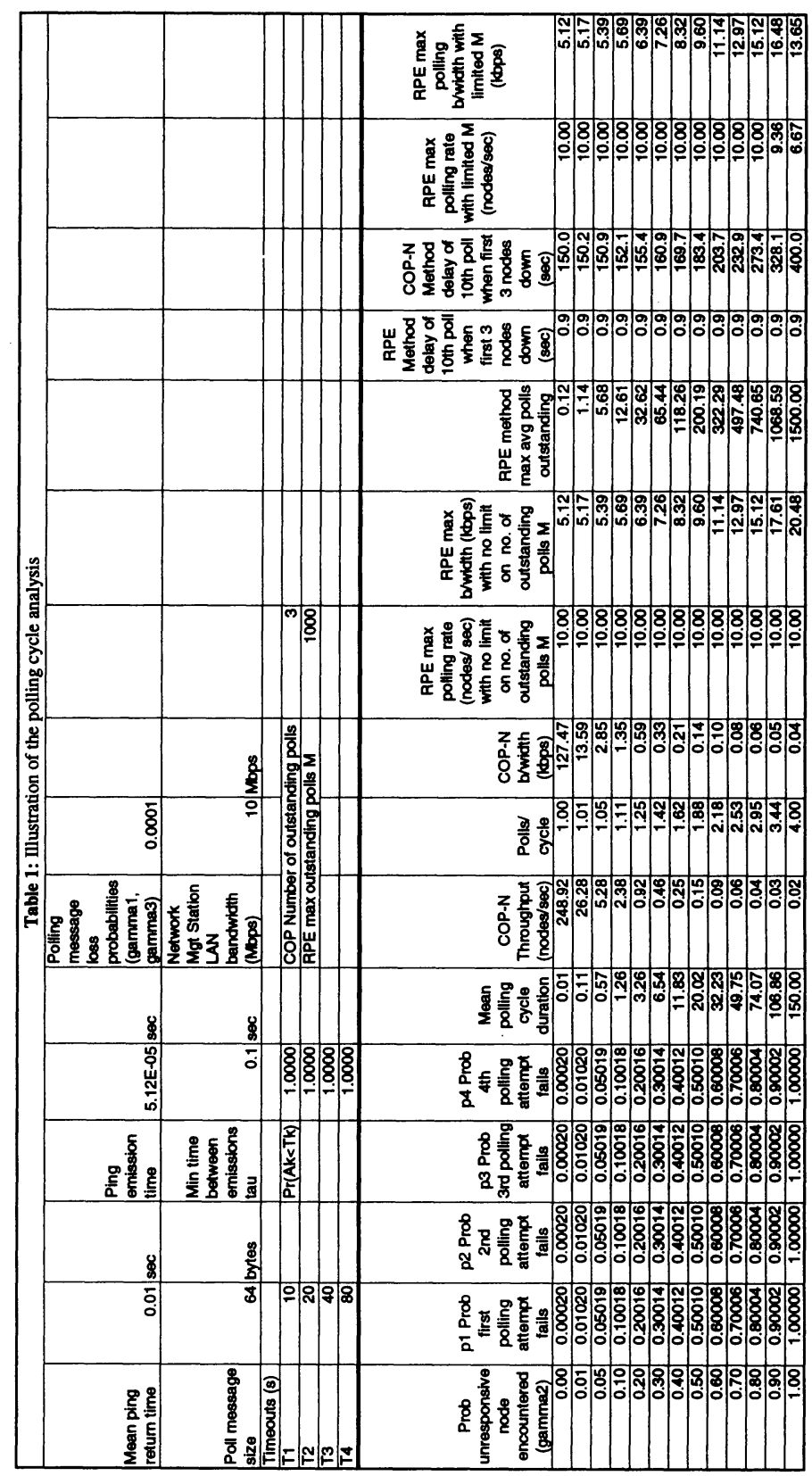



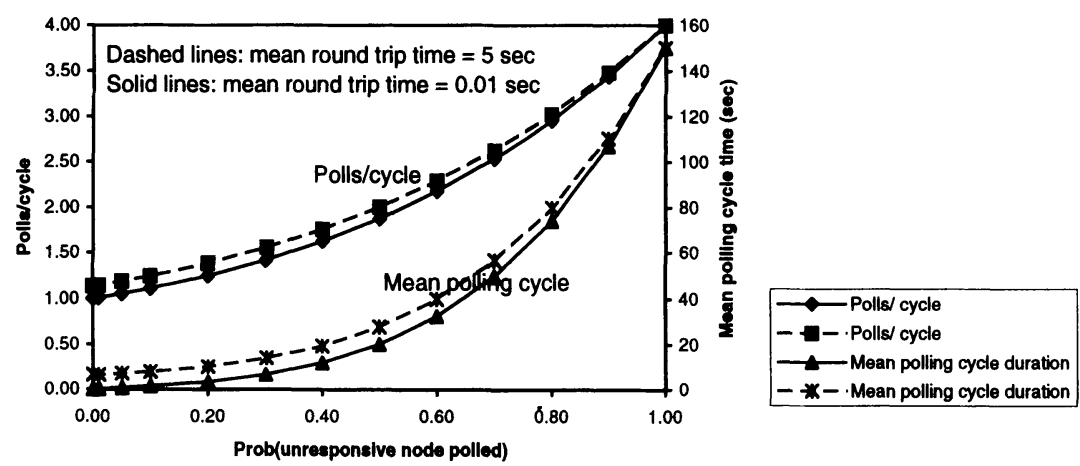

Figure 2 Polls per polling cycle (left axis), mean polling cycle duration (right axis).

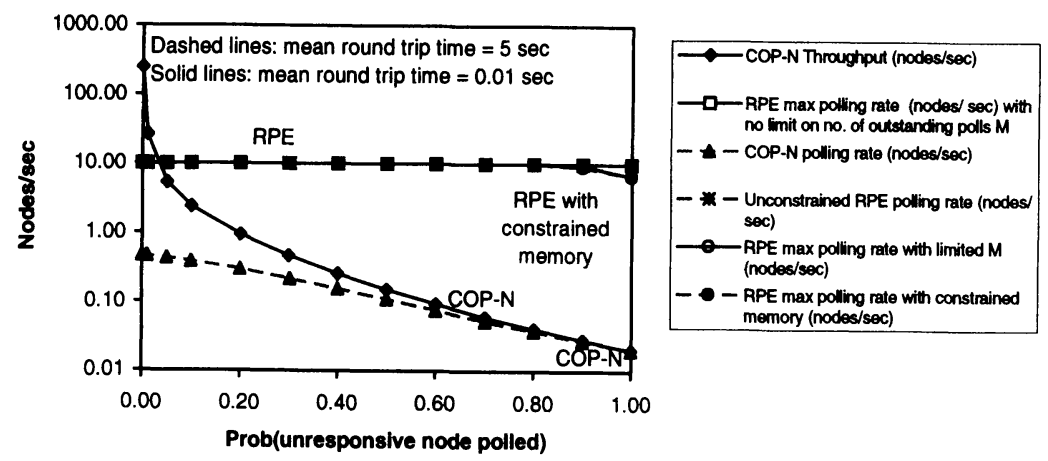

Figure 3 Nodes polled per second.

bandwidth usage is much lower under RPE than under COP-N. This means that RPE enables one to control the bandwidth required by polling much more tightly than can be done with COP-N. This is achieved by tuning $\tau$ until the required tradeoff between node polling rate and bandwidth usage is achieved.

Delay is illustrated in Figure 5. Under RPE, the delay to the first poll of the 10 th node with the first three nodes down is small and constant. Under COP-3, the delay to the first poll of the 10th node with the first three nodes down increases from a level slightly greater than the sum of the timeout intervals to a level that is considerably larger.

Finally, let us analyse the memory requirement to store unresolved outstanding polls under the RPE method. The average number of outstanding initiated polling cycles is equal to the mean polling cycle time multiplied by the maximum throughput. The average number of outstanding polls increases dramatically with the probability of encountering an inoperative node, as shown in Figure 6. To keep the node polling rate constant, the maximum number of nodes for which polls are 

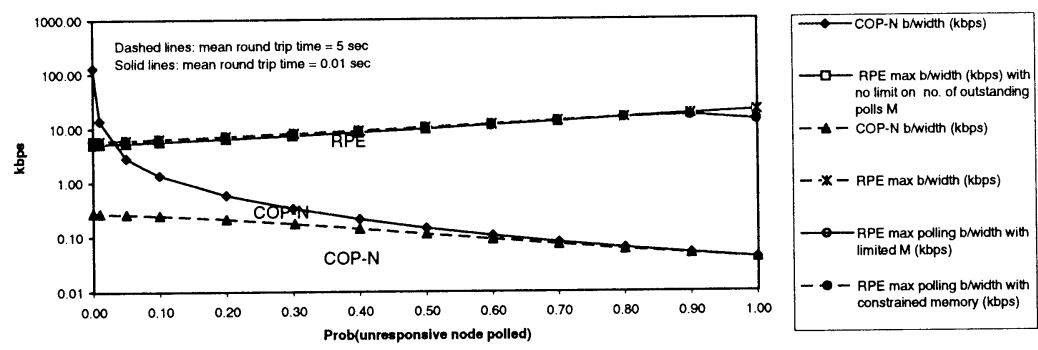

Figure 4 Bandwidth used by polling (kbps).

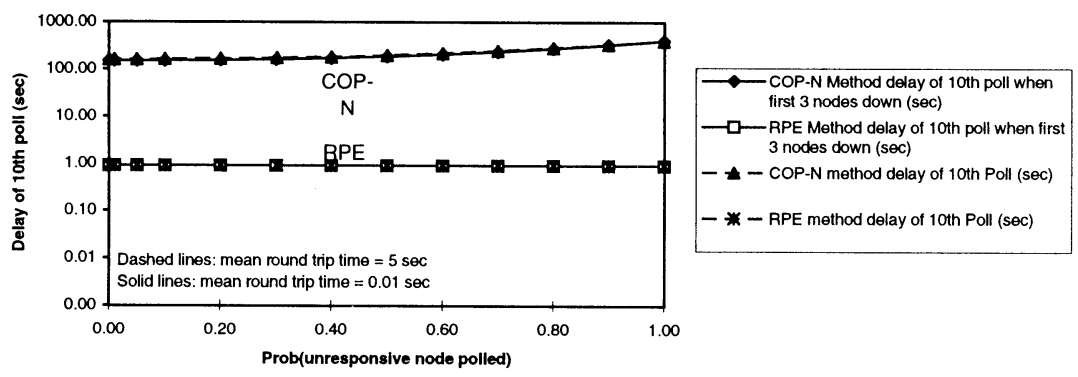

Figure 5 Delay of 10th poll (seconds).

unresolved, $M$, must be suitably large. However, if the probability of polling an unresponsive node, $\gamma_{2}$, is 0.5 or less, this number is not exorbitant, although it is sensitive to the round trip time. As $\gamma_{2}$ approaches one, the timeouts become the dominant factor in determining the average polling cycle time, and hence the average number of outstanding polls at maximum throughput. In our illustration, storage would be required for 1500 outstanding polling records. In practice, a high value of $\gamma_{2}$ would be undesirable, and perhaps indicative of a major problem in the managed network.

\subsection{Discussion}

Our analysis corroborates previously reported observations (Sturm, 1995) about the behaviour of COP-N. Let us first consider polling rates and polling bandwidth usage. COP-N cannot prevent surges of polling activity when ping return times (mean round trip times) are short. At the same time, polling activity all but ceases if the likelihood of polling an unresponsive node is high. By contrast, the proposed 


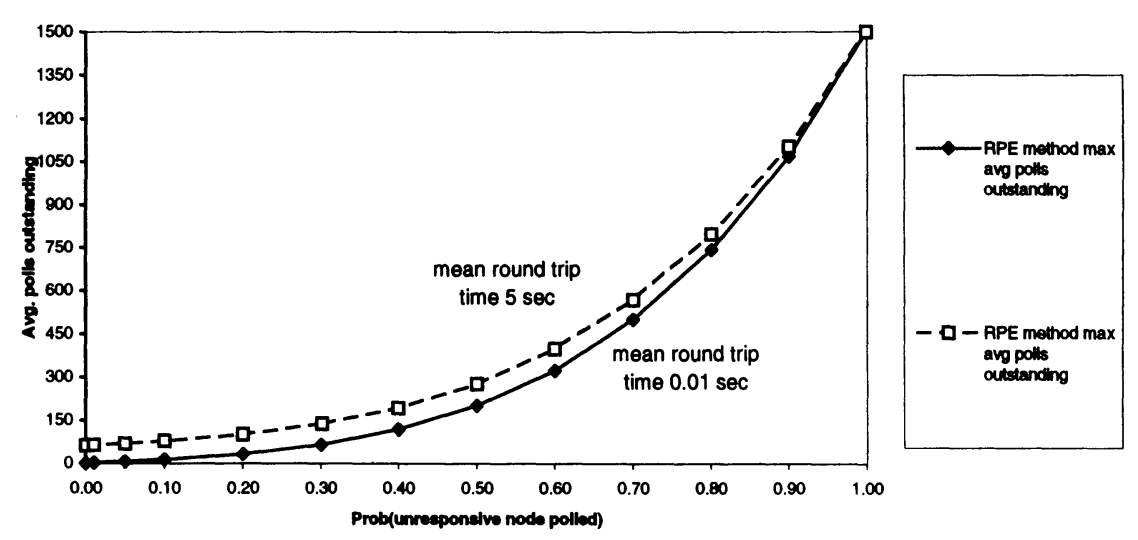

Figure 6 Average number of unresolved polls under RPE.

RPE method limits network polling bandwidth when ping round trip times are short and sustains it at a constant level when unresponsive nodes are encountered, provided that the network management system allows records for a sufficient number of outstanding polls to be stored. Thus, the RPE method may be tuned to limit polling bandwidth or sustain polling activity according to need. These points are highlighted in Figure 3 and Figure 4. With regard to polling delays, under COP-N, polling is effectively suspended until all timeouts associated with polls of unresponsive nodes have expired. On the other hand, RPE limits delays by ensuring that polls may be transmitted at regular intervals if needed, regardless of the state of the network.

\section{CONCLUSION}

We have presented RPE, a method for regulating the transmission of polls in a network management system that will sustain polling at a specified rate when multiple unresponsive nodes are encountered. Beneficial features of this method include (i) that it may be tuned to ensure that polls do not swamp the network with management traffic and (ii) that the node polling delay is insensitive to the state of the network. Hence, RPE overcomes some of the network management difficulties inherent in a previously implemented method, as described in the open literature.

\section{Acknowledgments}

This work was begun while the author was on assignment to AT\&T Global Information Solutions (AT\&T GIS) from AT\&T Bell Laboratories. He has benefited from discussions with Dennis Zavila and Steve Leonard of NCR (formerly AT\&T GIS), Gagan L. Choudhury and Kathleen Meier-Hellstern of AT\&T Laboratories (formerly AT\&T Bell Laboratories), and Alan Shepherd and Glen Shirey of Hewlett Packard. 


\section{REFERENCES}

AT\&T/HP (November 1994). AT\&T OneVision Node Manager Fundamentals for Network Operators (CW 9118-9000-1847). AT\&T/Hewlett Packard.

Eckberg, A. E. Jr., Luan, D. T., \& Lucantoni, D. M. (1990). An Approach to Controlling Congestion in ATM Networks. International Journal of Digital and Analog Communication Systems, 3, 199-209.

Rose, M. T. (1991). The Simple Book: An Introduction to Management of TCP/IP-based Internets. Englewood Cliffs: Prentice Hall.

Sturm, R. (March 1995). Q\&A. OpenView Advisor, 1(2), 11-13.

Sturm, R. (April 1995). Q\&A. OpenView Advisor, 1(3), 9-12.

\section{Biography}

André Bondi is a Senior Technical Staff Member in the Teletraffic and Performance Analysis Department at AT\&T Labs (formerly AT\&T Bell Laboratories). He has worked on a large variety of performance aspects of telecommunications, including network management, operator services, computer performance, ATM, call routing, and intelligent networking. Prior to joining AT\&T, he was an assistant professor of computer science at the University of California, Santa Barbara. Dr. Bondi holds a Ph.D. in computer science from Purdue University, an M.Sc. in statistics from University College London, and a B.Sc. in mathematics from the University of Exeter. 